\title{
Improved Prostate Cancer Biopsy Grading by Incorporation of Invasive Cribriform and Intraductal Carcinoma in the 2014 Grade Groups
}

\author{
Geert J.L.H. van Leenders ${ }^{a, *}$, Charlotte F. Kweldam ${ }^{a}$, Eva Hollemans ${ }^{a}$, Intan P. Kümmerlin ${ }^{a}$, \\ Daan Nieboer ${ }^{b, c}$, Esther I. Verhoef ${ }^{a}$, Sebastiaan Remmers ${ }^{b},{\text { Luca } \text { Incrocci }^{d} \text {, Chris H. Bangma }}^{b}$, \\ Theodorus H. van der Kwast ${ }^{e}$, Monique J. Roobol ${ }^{b}$ \\ ${ }^{a}$ Department of Pathology, Erasmus University Medical Centre, Rotterdam, The Netherlands; ${ }^{\mathrm{b}}$ Department of Urology, Erasmus University Medical Centre, \\ Rotterdam, The Netherlands; ${ }^{\mathrm{c}}$ Department of Public Health, Erasmus University Medical Centre, Rotterdam, The Netherlands; ${ }^{\mathrm{d}}$ Department of Radiotherapy, \\ Erasmus University Medical Centre, Rotterdam, The Netherlands; ${ }^{\mathrm{e}}$ Laboratory Medicine Program, University Health Network, Toronto, Canada
}

\section{Article info}

Article history:

Accepted July 31, 2019

Associate Editor:

Matthew Cooperberg

Statistical Editor:

Melissa Assel

Keywords:

Cribriform

Gleason score

Grade

Intraductal carcinoma

Prostate cancer

\begin{abstract}
Background: Grade groups (GGs) are an important parameter for therapeutic decision making in prostate cancer (Pca) patients. Invasive cribriform and/or intraductal carcinoma (CR/IDC) has an independent prognostic value for disease outcome, but are not included in the GG limiting their clinical use.

Objective: To perform a proof-of-principle study incorporating CR/IDC in the current GG. Design, setting, and participants: All prostate biopsies of 1031 men with screen-detected Pca between 1993 and 2000 were reviewed for the current GG (ranging from 1 to 5) and CR/IDC. The cribriform grade (cGrade) was equal to the GG if CR/IDC was present and GG minus 1 if not. GG1 was cGrade1 if intraductal carcinoma was absent.

Intervention: Biopsy review for GG and CR/IDC. A total of 406 patients had received radical prostatectomy (RP), 508 radiotherapy (RT), 108 surveillance, and eight hormonal therapy, and the treatment was unknown for one patient.

Outcome measurements and statistical analysis disease-specific survival (DSS), metastasisfree survival (MFS), and biochemical recurrence-free survival (BCRFS) after $15.1 \mathrm{yr}$ (interquartile range 10.9-19.7 yr) follow-up were compared using Harrell's C-statistic.

Results and limitations: The biopsy GGs were 486 GG1, 310 GG2, 104 GG3, 64 GG4, and 67 GG5; cGrade distributions were 738 cGrade1, 102 cGrade2, 91 cGrade3, 58 cGrade4, and 42 cGrade5. The cGrade had a better discriminative value than the GG for DSS (C-index 0.79; $95 \%$ confidence interval $0.74-0.83$ vs $0.76 ; 0.71-0.82)$ and MFS $(0.79 ; 0.74-0.84$ vs $0.77 ; 0.72-$ 0.82). The discriminative value for BCRFS after RP and RT was similar for both models. Different diagnostic, such as use of sextant biopsies, and therapeutic strategies in the 1990s are the limitations of this study.

Conclusions: The cGrade is a simple Pca grade modification with better discriminative values for DSS and MFS than the GG, particularly impacting decision making in men with current GG2 Pca.

Patient summary: Microscopic grading is an important factor for decision making in prostate cancer (Pca) patients. We show that a simple grade modification better predicts Pca outcome and might improve treatment choices.

(C) 2019 European Association of Urology. Published by Elsevier B.V. All rights reserved.

* Corresponding author. Department of Pathology, Erasmus University Medical Centre Rotterdam, Postbus 2040, 3000CA Rotterdam, The Netherlands. Tel. +31 10 7043901; Fax: +31 107038430. E-mail address: g.vanleenders@erasmusmc.nl (Geert J.L.H. van Leenders).
\end{abstract}

https://doi.org/10.1016/j.eururo.2019.07.051

0302-2838/৫ 2019 European Association of Urology. Published by Elsevier B.V. All rights reserved. 


\section{Introduction}

The Gleason grading system is one of the most important parameters for clinical decision making in prostate cancer (Pca) patients. Gleason score (GS) is entirely based on pathological assessment of tumour growth patterns and takes into account tumour heterogeneity. While the system was first developed in the late 1960s, it has been modified several times over the years and is currently based on the 2014 consensus conference of the International Society of Urologic Pathology (ISUP) [1,2].

Although the Gleason grading system has been established for several decades, it has a few weaknesses. Firstly, the GS ranges between 2 and 10, which might give patients with GS 6 a false impression to have a tumour of intermediate grade. Secondly, the clinically important distinction between GS $3+4$ and GS $4+3$ is not clearly reflected by GS 7 [3]. To overcome these weaknesses, Pierorazio and colleagues [4] introduced "grade groups" (GGs), which are defined as follows: GG1 (GS $\leq 6)$, GG2 (GS $3+4=7)$, GG3 (GS 4+3=7), GG4 (GS 8), and GG5 (GS9-10). The GGs were endorsed by the ISUP and the World Health Organization (WHO), and should be reported in conjunction with the GS $[1,2]$.

In recent years, much evidence was obtained that invasive cribriform and/or intraductal carcinoma (CR/IDC) are pathological features with strong independent predictive values for disease-specific survival (DSS), biochemical recurrence-free survival (BCRFS), and adverse pathological parameters at radical prostatectomy (RP) [5-10]. Patients with biopsy GG2 Pca with presence of CR/IDC show significantly shorter DSS and BCRFS than those without $[8,9]$. On the contrary, DSS and BCRFS of biopsy GG2 Pca patients without $\mathrm{CR} / \mathrm{IDC}$ are not statistically different from those of men with GG1 Pca $[8,9]$. Furthermore, patients with biopsy GG1 Pca with concomitant intraductal carcinoma often show aggressive features at subsequent RP with extraprostatic expansion in up to $64 \%$ of cases [11]. Although invasive cribriform Gleason pattern 4 and intraductal carcinoma are strictly two separate pathological entities, they show significant morphological overlap and often coincide. Despite the accumulating evidence of their additional prognostic impact, presence of CR/IDC is not included in the current grading system. This limits their comprehensive and simple incorporation in clinical decision models.

In the current proof-of-principle study, we investigate a modified Pca grading model for prostate biopsies-cribriform grade (cGrade)-which incorporates CR/IDC in the established GG system.

\section{Patients and methods}

\subsection{Patient selection}

We identified all 1078 men from the first screening round of the Dutch part of the European Randomized Study of Screening for Prostate Cancer (ERSPC), who had been diagnosed with Pca between November 1993 and March 2000 at the Erasmus University Medical Centre, Rotterdam,
The Netherlands [12]. The ERSPC is an ongoing multicentre randomised screening trial that was initiated in the early 1990s to evaluate the effect of screening with prostatespecific antigen (PSA) testing on disease-specific mortality rates. Exclusion criteria of the present study were unavailability of slides or paraffin blocks for review $(n=24)$ and presence of lymph node or distant metastasis at the time of diagnosis $(n=23)$, leaving 1031 patients for analysis. The study was approved by the institutional Medical Research Ethics Committee (MEC-2018-1614) and in concordance with the Declaration of Helsinki.

\subsection{Pathological evaluation}

Three investigators (C.K., I.K., and G.v.L.), who were blinded to patient information and outcome, revised all available histopathological slides $(n=1031)$ according to the 2014 WHO/ISUP recommendations [2]. For each biopsy core, we recorded GS, GG, presence of CR/IDC, and percentage Gleason pattern 4 [13]. Invasive cribriform structures were defined as both small and expansive malignant epithelial proliferations with intercellular lumina, in which the majority of tumour cells did not contact surrounding stroma and which spanned at least half of the glandular lumen. Intraductal carcinoma was diagnosed according to the WHO criteria [1]. Basal cell immunohistochemistry was performed to differentiate invasive cribriform from intraductal carcinoma in case this was not evident by histopathological parameters alone [9]. Since invasive cribriform and intraductal carcinoma show significant morphological overlap and both have added clinical values, we combined both patterns into one group (CR/IDC). The worst biopsy GG was used for statistical analyses.

\subsection{Cribriform grade}

The cGrade was conceived as a modification of the GG system and was determined as follows (Supplementary Fig. 1). For Pca patients with GG2-5, the cGrade is similar to the $\mathrm{GG}$ if CR/IDC is present. The cGrade is equal to the GG minus 1 , if CR/IDC is absent. For men with GG1, the cGrade is 1 . In the rare case of GG1 Pca with concomitant intraductal carcinoma, the assigned cGrade is 2 .

\subsection{Clinical follow-up}

After diagnosis and initial treatment, patients were monitored semiannually by a chart review to assess potential progression and secondary treatments. DSS was defined as the time from diagnosis to death attributed to Pca and was evaluated by an independent causes of death committee [12]. Metastasis-free survival (MFS) was defined as the time from diagnosis to the development of distant metastasis or censoring. Biochemical recurrence was defined as a PSA level of $\geq 0.2 \mathrm{ng} / \mathrm{ml}$ assessed at two consecutive time points $>3$ mo apart after RP or any PSA increase of $>2 \mathrm{ng} / \mathrm{ml}$ higher than the PSA nadir value after radiotherapy (RT) [14]. BCRFS was defined as the time from RP or the start of RT to biochemical recurrence or censoring. 
A

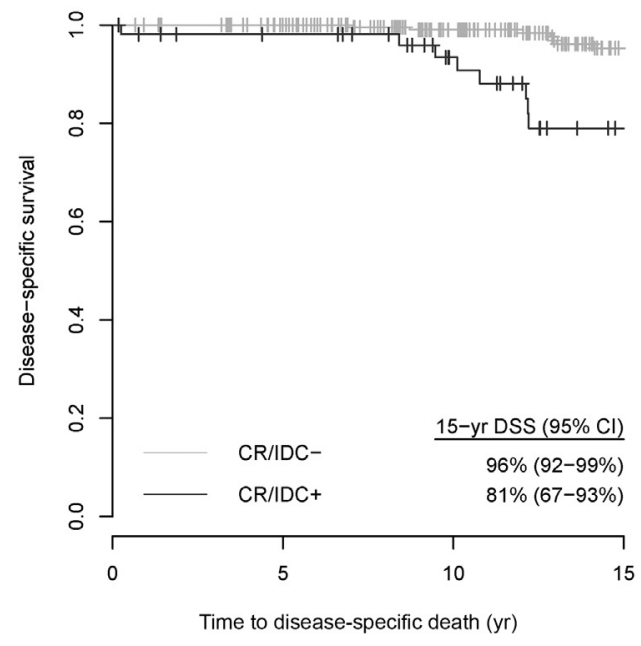

C

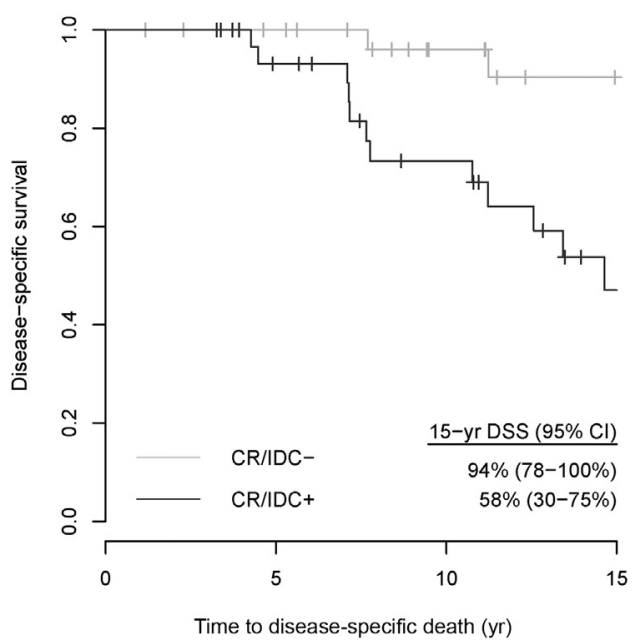

B

Grade group $3(n=104)$

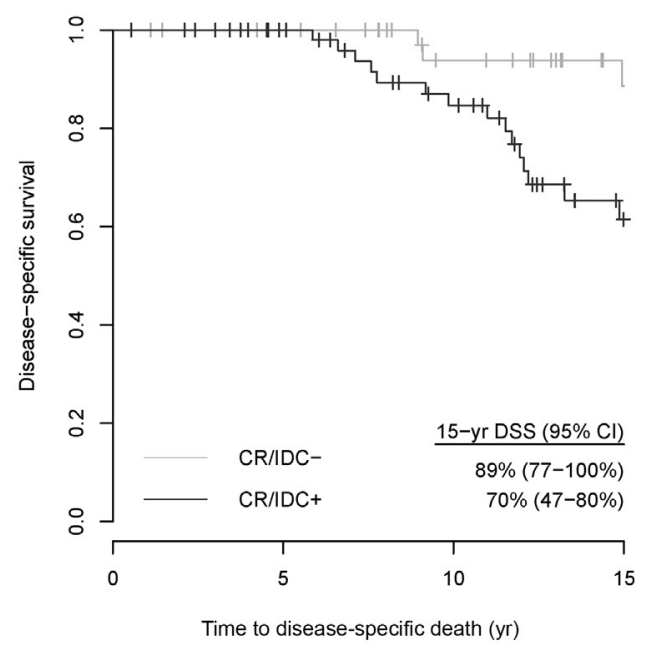

D

Grade group $5(n=67)$

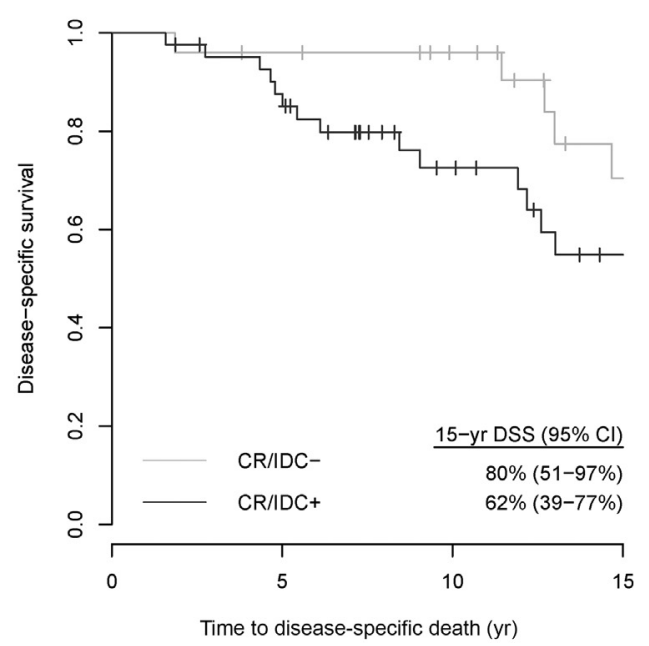

Fig. 1 - Kaplan-Meier survival probabilities of Pca patients stratified for the presence or absence of CR/IDC in (A) grade group (GG) 2, (B) GG3, (C) GG4, and (D) GG5.

$\mathrm{CI}=$ confidence interval; $\mathrm{CR} / \mathrm{IDC}=$ invasive cribriform and/or intraductal carcinoma; $\mathrm{DSS}=$ disease-specific survival; Pca = prostate cancer.

\subsection{Statistical analysis}

For DSS and BCRFS analyses, both GG and cGrade were included in five categories (GG1-5 and cGrade1-5), and percentage Gleason pattern 4 was included as a continuous variable. Hazard ratios (HRs) for survival time were calculated using univariable Cox proportional hazard regression. To adjust for different treatments, we included a stratified baseline hazard for treatment and estimated common HR for GG and cGrade. Survival probabilities were plotted using Kaplan-Meier curves. Discriminative ability for grading models was quantified using Harrell's C-index. All statistical analyses were performed in SPSS version 24 (IBM, Chicago, IL, USA) and R version 3.2.2 (R, Vienna,
Austria). Two-sided $p$ values of $<0.05$ were considered statistically significant.

\section{Results}

\subsection{Patient characteristics}

Our study cohort contained 1031 screen-detected Pca patients (Table 1). Their median age at the time of diagnosis was $67 \mathrm{yr}$ (interquartile range [IQR] 62-71 yr) and the median PSA level was $5.6 \mathrm{ng} / \mathrm{ml}$ (IQR 3.9-8.8 ng/ml). In total, 549 (53.3\%) men received six biopsies, 460 (44.6\%) seven biopsies, and 22 (2.1\%) eight biopsies. Four hundred and six (39.2\%) patients underwent RP, 508 (49.0\%) underwent RT, 
Table 1 - Patient characteristics of the study population.

\begin{tabular}{|c|c|c|c|c|c|}
\hline & $\begin{array}{c}\mathrm{GS} \leq 6 \mathrm{GG} 1 \\
(n=486)\end{array}$ & $\begin{array}{c}\text { GS } 3+4=7 \text { GG2 } \\
(n=310)\end{array}$ & $\begin{array}{c}\text { GS } 4+3=7 \text { GG3 } \\
(n=103)\end{array}$ & $\begin{array}{c}\text { GS } 8 \text { GG4 } \\
(n=64)\end{array}$ & $\begin{array}{c}\text { GS 9-10 GG5 } \\
(n=67)\end{array}$ \\
\hline Age, median (IQR) & $66(61-70)$ & $67(62-71)$ & $69(65-72)$ & $69(66-72)$ & $67(64-71)$ \\
\hline PSA, median (IQR) & $4.7(3.5-6.9)$ & $5.8(4.0-9.0)$ & $7.4(4.7-14.9)$ & $11.0(6.1-17.4)$ & $9.4(5.3-16.3)$ \\
\hline CR/IDC, $n$ (\%) & $4(0.8)$ & $54(17.4)$ & $60(57.7)$ & $33(51.6)$ & $42(62.7)$ \\
\hline Radical prostatectomy, $n$ (\%) & $216(44.4)$ & $129(41.6)$ & $33(32.0)$ & $14(21.9)$ & $14(20.9)$ \\
\hline Radiation therapy, $n$ (\%) & $188(38.7)$ & $154(49.7)$ & $66(64.1)$ & $48(75.0)$ & $52(77.6)$ \\
\hline Active surveillance/WW, $n$ (\%) & $80(16.5)$ & $23(7.4)$ & $3(2.9)$ & $1(1.6)$ & $1(1.5)$ \\
\hline Hormonal therapy, $n(\%)$ & $2(0.4)$ & $3(1.0)$ & $2(1.9)$ & $1(1.6)$ & 0 \\
\hline Unknown, $n(\%)$ & 0 & $1(0.3)$ & 0 & 0 & 0 \\
\hline
\end{tabular}

108 (10.5\%) were under watchful waiting/active surveillance, and eight $(0.8 \%)$ received hormonal therapy. Primary treatment was unknown in one $(0.1 \%)$ patient. The median follow-up of patients being alive at the last follow-up was $15.1 \mathrm{yr}$ (IQR 10.9-19.7 yr).

The biopsy grade distribution was as follows: 486 Pca cases were graded as GG1 (GS $\leq 6), 310$ cases as GG2 (GS $3+4=7$ ), 104 cases as GG3 (GS $4+3=7$ ), 64 cases as GG4 (GS 8 ), and 67 cases as GG5 (GS 9 or 10). A total of $566(54.9 \%)$ men died during follow-up, 90 (8.7\%) of whom died from Pca. Disease-specific death occurred in 2.1\% (10/486) of patients diagnosed with biopsy GG1, 6.5\% (20/310) with GG2, 22.1\% (23/104) with GG3, 25.0\% (16/64) with GG4, and 31.3\% (21/67) with GG5 Pca.

\subsection{Cribriform growth and/or intraductal carcinoma and DSS}

Four $(0.8 \%)$ GG1 $(G S \leq 6)$ Pca patients had concomitant intraductal carcinoma of the prostate. CR/IDC was observed in 54 (17.4\%), 60 (57.7\%), 33 (51.6\%), and 42 (62.7\%) patients with GG2, GG3, GG4, and GG5 Pca, respectively (Fig. 1). In multivariable analysis including the five GG categories, biopsy CR/IDC had additional predictive values for DSS (HR 3.8; 95\% confidence interval $[\mathrm{CI}] 2.3-6.3 ; p<0.001)$ and MFS (HR 3.7; 95\% CI 2.2-6.2; $p<0.001$ ). Adding an interaction between GG and CR/IDC did not lead to a significantly better model fit for DSS $(p=0.8)$ or MFS $(p=$ 0.6). Of interest, DSS of men with biopsy GG2 Pca without CR/IDC was not different from the DSS of those with GG1 Pca (HR 1.9; 95\% CI 0.8-4.7; $p=0.15$ ). While percentage Gleason pattern 4 has also been recognised as a parameter for clinical stratification of GG2 Pca patients, it did not have a predictive value for DSS (HR 1.5; 95\% CI 0.03-73.7; $p=0.8$ ) or MFS (HR 1.4; 95\% CI 0.03-64.1; $p=0.9$ ) as a continuous variable in our cohort $[1,5,15]$.

\subsection{Cribriform grade modification}

Distribution of cGrade among the 1031 patients was as follows: 738 patients were graded as having cGrade1, 102 as cGrade2, 91 as cGrade3, 58 as cGrade4, and 42 as cGrade5 Pca (Table 2). Incorporation of CR/IDC in the cGrade had the most prominent effect in GG2 tumours, of which 256/310 (82.6\%) were classified as cGrade1. Reclassification occurred
Table 2 - Distribution and relation of grade groups (GGs) and cribriform grade (cGrade).

\begin{tabular}{lllllll}
\hline & GG1 & GG2 & GG3 & GG4 & GG5 & Total \\
\hline cGrade1 & 482 & 256 & - & - & - & 738 \\
cGrade2 & 4 & 54 & 44 & - & - & 102 \\
cGrade3 & - & - & 60 & 31 & - & 91 \\
cGrade4 & - & - & - & 33 & 25 & 58 \\
cGrade5 & - & - & - & - & 42 & 42 \\
Total & 486 & 310 & 104 & 64 & 67 & 1031 \\
\hline
\end{tabular}

in $42.3 \%$ (44/104), 48.4\% (31/64), and 37.3\% (25/67) of GG3, GG4, and GG5 Pca cases, respectively. Only four out of 486 patients with GG1 Pca $(0.8 \%)$ were upgraded to cGG2 due to the presence of intraductal carcinoma.

\subsection{DSS and MFS}

Both grading systems were strongly associated with clinical outcome (Table 3). The discriminative value of the cGrade (C-index 0.79 ; 95\% CI 0.74-0.83) in predicting DSS was significantly ( $p=0.029$ ) higher than that of the GG (C-index 0.76; 95\% CI 0.71-0.82; Fig. 2). The cGrade model (C-index 0.79; 95\% CI 0.74-0.84) also showed better discrimination than the GG model (C-index 0.77; 95\% CI 0.72-0.82) for MFS, although this did not meet conventional measures of statistical significance $(p=0.062)$.

\subsection{Biochemical recurrence-free survival}

Of the 406 patients who had undergone RP, 216 (53\%) had GG1, 129 (32\%) GG2, 33 (8\%) GG3, 14 (3\%) GG4, and 14 (3\%) GG5 Pca. Conversion to the proposed cGrade distribution resulted in 326 patients (80\%) having cGrade1, 34 (8\%) cGrade2, 27 (7\%) cGrade3, 12 (3\%) cGrade4, and seven (2\%) cGrade5 Pca. In total, 85 (20.9\%) patients experienced biochemical recurrence after a median of $4.5 \mathrm{yr}$ (IQR 2.3$9.3 \mathrm{yr}$ ). Both grading models were strongly associated with postoperative BCRFS (Table 4). There was no significant difference $(p=0.4)$ between the discriminative values of the cGrade model (C-index 0.65; 95\% CI 0.58-0.71) and the GG model (C-index 0.65; 95\% CI 0.59-0.71).

In total, 223 out of 508 patients who received RT (43.9\%) experienced biochemical recurrence after a median 
A

Grade group

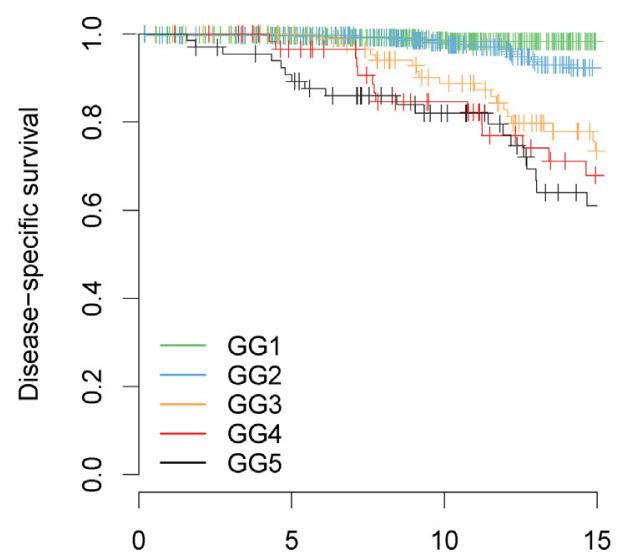

Time to disease-specific death (yr)

No. at risk

GG1 486

GG2 310

GG3 104

GG4

GG5

64
67

446
284
90
54
58

345
212
65
36
38

193
122
32
20
20

B

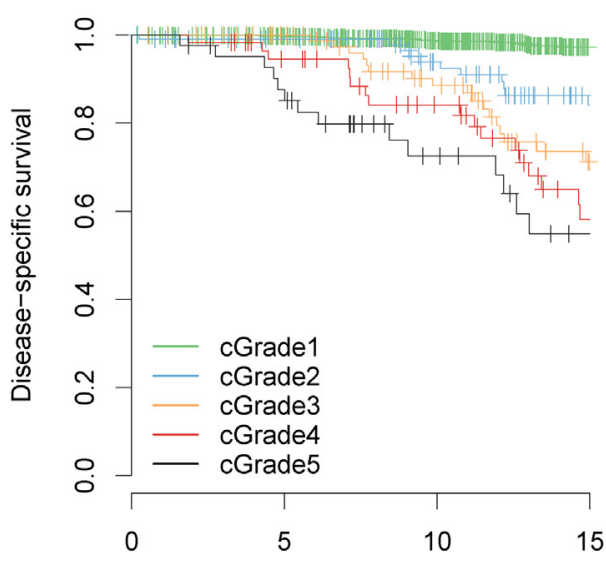

Time to disease-specific death $(y r)$

No. at risk

$\begin{array}{lllll}\text { cGrade1 } & 738 & 679 & 520 & 294\end{array}$

$\begin{array}{lllll}\text { cGrade2 } & 102 & 91 & 66 & 38\end{array}$

$\begin{array}{lllll}\text { cGrade3 } & 91 & 78 & 55 & 28\end{array}$

$\begin{array}{lllll}\text { cGrade4 } & 58 & 49 & 36 & 17\end{array}$

$\begin{array}{lllll}\text { cGrade5 } & 42 & 35 & 19 & 10\end{array}$

Fig. 2 - Kaplan-Meier survival probabilities of Pca patients graded according to the (A) current grade group model and (B) proposed cGrade model. cGrade = cribriform grade; $G G=$ grade group; $P c a=$ prostate cancer.

Table 3 - Cox proportional hazard models for prostate cancer disease-specific survival and metastasis-free survival stratified by grade groups (GGs) and cribriform grade (cGrade).

\begin{tabular}{|c|c|c|c|c|c|c|c|c|}
\hline \multirow{3}{*}{ Group } & \multicolumn{4}{|c|}{ Disease-specific survival } & \multicolumn{4}{|c|}{ Metastasis-free survival } \\
\hline & \multicolumn{2}{|c|}{ Grade group } & \multicolumn{2}{|c|}{ cGrade } & \multicolumn{2}{|c|}{ Grade group } & \multicolumn{2}{|c|}{ cGrade } \\
\hline & HR (95\% CI) & $p$ value & HR (95\% CI) & $p$ value & HR (95\% CI) & $p$ value & HR (95\% CI) & $p$ value \\
\hline 1 & ref & - & ref & - & ref & - & ref & - \\
\hline 2 & $3.1(1.4-6.6)$ & 0.003 & $4.6(2.3-9.1)$ & $<0.001$ & $3.8(1.7-8.4)$ & $<0.001$ & $3.7(1.8-7.7)$ & $<0.001$ \\
\hline 3 & $10.3(4.8-22.2)$ & $<0.001$ & $8.4(4.5-15.8)$ & $<0.001$ & $10.4(4.6-23.3)$ & $<0.001$ & $9.3(5.0-17.2)$ & $<0.001$ \\
\hline 4 & $11.9(5.3-26.7)$ & $<0.001$ & $11.2(5.9-21.4)$ & $<0.001$ & $16.7(7.3-38.3)$ & $<0.001$ & $12.1(6.4-23.0)$ & $<0.001$ \\
\hline 5 & $16.4(7.5-35.8)$ & $<0.001$ & $17.9(9.1-35.2)$ & $<0.001$ & $18.0(7.9-40.9)$ & $<0.001$ & $15.6(7.8-31.2)$ & $<0.001$ \\
\hline
\end{tabular}

Table 4 - Cox proportional hazard models for biochemical recurrence-free survival after radical prostatectomy and radiation therapy stratified by Grade groups (GG) and cribriform Grade (cGrade).

\begin{tabular}{|c|c|c|c|c|c|c|c|c|}
\hline \multirow{3}{*}{ Group } & \multicolumn{4}{|c|}{ BCRFS after radical prostatectomy } & \multicolumn{4}{|c|}{ BCRFS after radiation therapy } \\
\hline & \multicolumn{2}{|c|}{ Grade group } & \multicolumn{2}{|c|}{ cGrade } & \multicolumn{2}{|c|}{ Grade group } & \multicolumn{2}{|c|}{ cGrade } \\
\hline & $\mathrm{HR}(95 \% \mathrm{CI})$ & $p$ value & $\mathrm{HR}(95 \% \mathrm{CI})$ & $p$ value & $\mathrm{HR}(95 \% \mathrm{CI})$ & $p$ value & $\mathrm{HR}(95 \% \mathrm{CI})$ & $p$ value \\
\hline 1 & ref & - & ref & - & ref & - & ref & - \\
\hline 2 & $2.1(1.2-3.5)$ & 0.006 & $2.2(1.1-4.4)$ & 0.02 & $1.9(1.3-2.8)$ & 0.001 & $2.5(1.7-3.7)$ & $<0.001$ \\
\hline 3 & $4.1(2.1-7.8)$ & $<0.001$ & $4.4(2.4-7.9)$ & $<0.001$ & $4.4(2.9-6.7)$ & $<0.001$ & $4.4(3.0-6.5)$ & $<0.001$ \\
\hline 4 & $5.0(2.2-11.6)$ & $<0.001$ & $4.5(1.9-10.4)$ & 0.001 & $5.9(3.8-9.3)$ & $<0.001$ & $5.7(3.8-8.5)$ & $<0.001$ \\
\hline 5 & $5.2(2.2-11.9)$ & $<0.001$ & $4.4(1.6-12.2)$ & 0.005 & $7.5(4.9-11.5)$ & $<0.001$ & $7.4(4.8-11.5)$ & $<0.001$ \\
\hline
\end{tabular}

follow-up of $4.8 \mathrm{yr}$ (IQR 2.7-8.2 yr). According to the current grading system, 188 (37\%) men had GG1, 154 (30\%) GG2, 66 (13\%) GG3, 48 (9\%) GG4, and 52 (10\%) GG5 Pca, corresponding to 306 (60\%) cGrade1, 64 (13\%) cGrade2, 59 (12\%)
cGrade3, 44 (9\%) cGrade4, and 35 (7\%) cGrade5 (Table 4). The biopsy cGrade (C-index $0.71 ; 95 \%$ CI $0.68-0.75$ ) had a comparable discriminative value $(p=0.2)$ for BCRFS after RT with the GG (C-index 0.72; 95\% CI 0.69-0.75). 


\subsection{Eligibility for active surveillance}

The most prominent effect of the cGrade model over the current GG model is the reclassification of men potentially being eligible for active surveillance. In total 272/1031 (26.4\%) men fulfilled the Prostate Cancer Research International Active Surveillance (PRIAS) criteria for active surveillance (GG1, positive biopsies $\leq 2$, $\leq \mathrm{cT} 2$, PSA $<10$ $\mathrm{ng} / \mathrm{ml}$, and PSA density $<0.2 \mathrm{ng} / \mathrm{ml}^{2}$ ). In case cGrade 1 was used instead of GG1, the total number of patients fulfilling the PRIAS criteria increased by 84 (8.1\%) to 356/1031 (34.5\%) of the entire population. The effect was larger if less stringent eligibility criteria were applied. If the number of positive biopsies was not taken into account, as increasingly happens in current practice with larger numbers of systematic as well as multiparametric magnetic resonance imaging (mpMRI)-targeted biopsies, the total number of patients being eligible for active surveillance increased by 151 (14.6\%), from 361 (35.0\%) for GG1 to 512 (49.7\%) for cGrade1.

\section{Discussion}

Pathological grading by GS and GG is one of the most important parameters for predicting Pca outcome and guiding clinical decision making. In the past decade, intraductal carcinoma and invasive cribriform carcinoma have both been recognised as novel pathological features with independent predictive values for adverse Pca outcome. Incorporation of these parameters in Pca grading is, however, needed to facilitate widespread implementation into clinical practice. In the current study, we demonstrate that a simple modification of the GG system with respect to the presence or absence of CR/IDC outperforms current Pca grading. In a set of over a thousand diagnostic prostate biopsies, we found that the cGrade showed a stronger discriminative value for the prediction of DSS and MFS than contemporary Pca grading.

In the past decade, the independent predictive value of additional histopathological features has been recognised. Intraductal carcinoma is a proliferation of malignant cells in pre-existent prostate glands, which has been related to an adverse disease outcome but which is not included in Gleason grading or the GG $[10,11,13,16]$. Invasive cribriform growth is a Gleason pattern 4 subgroup associated with worse DSS than the other Gleason 4 growth patterns $[5,6,9,17,18]$. Invasive cribriform and intraductal carcinoma show overlapping morphological features, and might be difficult to differentiate without the use of basal cell immunohistochemistry. Therefore, both patterns are often grouped as one entity [13,19]. Percentage Gleason pattern 4 has also been shown to have an additive prognostic value for Pca outcome $[5,15]$. However, we did not find any additive predictive value of Gleason pattern 4 . This discordance might be explained by our analysis of percentage Gleason pattern 4 as a continuous instead of a dichotomised variable.

One of the most important weaknesses of Gleason grading is its substantial interobserver variability [20]. While interob- server variability might have minor therapeutic implications in patients with GG3-5 Pca, it has significant impact on men with GG1 and GG2 disease since their distinction commonly determines eligibility for active surveillance. Grading discordances particularly occur in differentiating poorly formed and fused Gleason pattern 4 from tangentially sectioned pattern 3 [20,21]. Interobserver agreement is, however, excellent for cribriform pattern 4 , which is generally not confused with Gleason pattern 3 [20,21]. The fact that men with biopsy GG2 Pca without CR/IDC had comparable clinical outcome to those with GG1 disease, and the existence of substantial interobserver variability in distinguishing between GG1 and GG2 Pca constitute an important rationale for coalescing both groups into cGrade1 $[8,9]$. This is also supported by the low rate of lymph node metastases in men with GG2 Pca. Diolombi and Epstein [22] found 0.6\% pelvic lymph node metastasis among 3235 men with GG2 Pca at RP without stratification for CR/IDC. In another GG2 RP cohort, lymph node metastasis were present in 12/228 (5.3\%) patients with CR/IDC but in none of 192 men without CR/IDC [23]. Studies comparing the interobserver variability of both models, however, still have to be performed.

In the current study, 256 out of 310 men with biopsy GG2 disease were reclassified as cGrade1. The proportion of men fulfilling the PRIAS criteria for active surveillance increased from $26.4 \%$ to $34.5 \%$ if cGrade 1 was used instead of GG1. This number further increased if less stringent eligibility criteria were used. Since upgrading is the most important parameter for surveillance discontinuation, applying the cGrade instead of GG would lead not only to a larger number of patients meeting the inclusion criteria, but also to fewer dropouts due to grade progression from GG1 to GG2 [24].

Gleason grading has been a work in progress for more than half a century. The WHO/ISUP significantly modified the Gleason grading system in 2005 and achieved minor modifications in $2014[2,25]$. While the cGrade model outperformed the current GG model, we feel that there is still room for improvement. For instance, we noted in our cohort that men with GG4 disease without CR/IDC had DSS rates comparable to those with GG2 and GG3 disease. This is in line with the findings of Harding-Jackson et al [27], who also found relatively good outcome in GG4 Pca patients without cribriform pattern. With the introduction of the GG system, all patients with GS8 are classified as GG4 Pca patients irrespective of the GS being $3+5,4+4$, or $5+3$. Van den Bergh et al found that men with GS $3+5=8$ had significantly better BCRFS than GS8 patients with any of the other grade combinations, although such a difference could not be confirmed by others [26-28]. Detailed analyses for the presence of $\mathrm{CR} / \mathrm{IDC}$ in this heterogeneous population of GG4 Pca patients might further improve Pca grading. At this moment, we do not advocate to modify Pca grading. Additional improvements, reproducibility studies, and independent validations are warranted before grade modifications can be implemented with broad support of the scientific community.

The strongest point of this study is the use of a wellcharacterised population of Pca patients with long-term follow-up and strong clinical endpoints. The diagnostic and 
treatment modalities in the 1990s, however, were significantly different from contemporary strategies that include a larger number of biopsies, application of mpMRI, and active surveillance. Therefore, validation of the current findings in more contemporary cohorts is important before its routine adoption. We expect that the performance of the cGrade model will be even better in contemporary cohorts, since a larger number of systematic and targeted biopsies decrease biopsy sampling artefacts.

\section{Conclusions}

In this proof-of-principle study, we demonstrate that the biopsy cGrade is a simple modification of the GG system having better discriminative values for DSS and MFS than current Pca grading. Reclassification of GG2 Pca patients with favourable outcome as cGrade1 might allow more patients to be considered for active surveillance.

Author contributions: Geert J.L.H. van Leenders had full access to all the data in the study and takes responsibility for the integrity of the data and the accuracy of the data analysis.

Study concept and design: van Leenders, Roobol.

Acquisition of data: Kweldam, Kümmerlin, van Leenders, van der Kwast. Analysis and interpretation of data: van Leenders, Nieboer, Incrocci, Bangma, van der Kwast, Roobol.

Drafting of the manuscript: van Leenders.

Critical revision of the manuscript for important intellectual content: van Leenders, Kweldam, Hollemans, Kümmerlin, Nieboer, Verhoef, Remmers, Incrocci, Bangma, van der Kwast, Roobol.

Statistical analysis: van Leenders, Nieboer.

Obtaining funding: None.

Administrative, technical, or material support: Hollemans, Verhoef, Remmers.

Supervision: van Leenders, Roobol.

Other: None.

Financial disclosures: Geert J.L.H. van Leenders certifies that all conflicts of interest, including specific financial interests and relationships and affiliations relevant to the subject matter or materials discussed in the manuscript (eg, employment/affiliation, grants or funding, consultancies, honoraria, stock ownership or options, expert testimony, royalties, or patents filed, received, or pending), are the following: None.

Funding/Support and role of the sponsor: None.

Conflicts of interest: The authors have nothing to disclose.

\section{Appendix A. Supplementary data}

Supplementary material related to this article can be found, in the online version, at doi:https://doi.org/10.1016/j. eururo.2019.07.051.

\section{References}

[1] Moch H, Humphrey PA, Ulbright TM, Reuter VE. WHO classification of tumours of the urinary system and male genital organs. Lyon, France: International Agency for Research on Cancer (IARC); 2016. p. $135-67$.
[2] Epstein JI, Egevad L, Amin MB, et al. The 2014 International Society of Urological Pathology (ISUP) consensus conference on Gleason grading of prostatic carcinoma: definition of grading patterns and proposal for a new grading system. Am J Surg Pathol (40):2016;244-52.

[3] Stark JR, Perner S, Stampfer MJ, et al. Gleason score and lethal prostate cancer: does $3+4=4+3$ ? J Clin Oncol 2009;27:3459-64.

[4] Pierorazio PM, Walsh PC, Partin AW, Epstein JI. Prognostic Gleason grade grouping: data based on the modified Gleason scoring system. BJU Int 2013;111:753-60.

[5] Choy B, Pearce SM, Anderson BB, et al. Prognostic significance of percentage and architectural types of contemporary Gleason pattern 4 prostate cancer in radical prostatectomy. Am J Surg Pathol 2016;40:1400-6.

[6] Dong F, Yang P, Wang C, et al. Architectural heterogeneity and cribriform pattern predict adverse clinical outcome for Gleason grade 4 prostatic adenocarcinoma. Am J Surg Pathol 2013;37:1855-61.

[7] Iczkowski KA, Paner GP, Van der Kwast T. The new realization about cribriform prostate cancer. Adv Anat Pathol 2018;25:31-7.

[8] Kweldam CF, Kummerlin IP, Nieboer D, et al. Prostate cancer outcomes of men with biopsy Gleason score 6 and 7 without cribriform or intraductal carcinoma. Eur J Cancer 2016;66:26-33.

[9] Kweldam CF, Kummerlin IP, Nieboer D, et al. Disease-specific survival of patients with invasive cribriform and intraductal prostate cancer at diagnostic biopsy. Mod Pathol 2016;66:26-33.

[10] Van der Kwast T, Al Daoud N, Collette L, et al. Biopsy diagnosis of intraductal carcinoma is prognostic in intermediate and high risk prostate cancer patients treated by radiotherapy. Eur J Cancer 2012;48:1318-25.

[11] Khani F, Epstein JI. Prostate biopsy specimens with Gleason 3+3=6 and intraductal carcinoma: radical prostatectomy findings and clinical outcomes. Am J Surg Pathol 2015;39:1383-9.

[12] Schroder FH, Hugosson J, Roobol MJ, et al. Screening and prostatecancer mortality in a randomized European study. N Engl J Med 2009;360:1320-8.

[13] Kweldam CF, Wildhagen MF, Steyerberg EW, Bangma CH, van der Kwast TH, van Leenders GJ. Cribriform growth is highly predictive for postoperative metastasis and disease-specific death in Gleason score 7 prostate cancer. Mod Pathol 2015;28:457-64.

[14] Roach 3rd M, Hanks G, Thames Jr H, et al. Defining biochemical failure following radiotherapy with or without hormonal therapy in men with clinically localized prostate cancer: recommendations of the RTOG-ASTRO Phoenix Consensus Conference. Int J Radiat Oncol Biol Phys 2006;65:965-74.

[15] Sauter G, Steurer S, Clauditz TS, et al. Clinical utility of quantitative Gleason grading in prostate biopsies and prostatectomy specimens. Eur Urol 2016;69:592-8.

[16] Guo CC, Epstein JI. Intraductal carcinoma of the prostate on needle biopsy: histologic features and clinical significance. Mod Pathol 2006;19:1528-35.

[17] Iczkowski KA, Torkko KC, Kotnis GR, et al. Digital quantification of five high-grade prostate cancer patterns, including the cribriform pattern, and their association with adverse outcome. Am J Clin Pathol 2011;136:98-107.

[18] Trudel D, Downes MR, Sykes J, Kron KJ, Trachtenberg J, van der Kwast TH. Prognostic impact of intraductal carcinoma and large cribriform carcinoma architecture after prostatectomy in a contemporary cohort. Eur J Cancer 2014;50:1610-6.

[19] Chua MLK, Lo W, Pintilie M, et al. A prostate cancer "nimbosus": genomic instability and SChLAP1 dysregulation underpin aggression of intraductal and cribriform subpathologies. Eur Urol 2017;72:665-74.

[20] Egevad L, Ahmad AS, Algaba F, et al. Standardization of Gleason grading among 337 European pathologists. Histopathology 2013;62:247-56. 
[21] Kweldam CF, Nieboer D, Algaba F, et al. Gleason grade 4 prostate adenocarcinoma patterns: an inter-observer agreement study among genitourinary pathologists. Histopathology 2016;69:441-9.

[22] Diolombi ML, Epstein JI. Metastatic potential to regional lymph nodes with Gleason score $<\mid=7$, including tertiary pattern 5 , at radical prostatectomy. BJU Int 2017;119:872-8.

[23] Hollemans E, Verhoef EI, Bangma $\mathrm{CH}$, et al. Large cribriform growth pattern identifies ISUP grade 2 prostate cancer at high risk for recurrence and metastasis. Mod Pathol 2019;32:139-46.

[24] Simpkin AJ, Tilling K, Martin RM, et al. Systematic review and metaanalysis of factors determining change to radical treatment in active surveillance for localized prostate cancer. Eur Urol 2015;67:993-1005.

[25] Epstein JI, Allsbrook Jr WC, Amin MB, Egevad LL, Committee ISUP Grading. The 2005 International Society of Urological Pathology
(ISUP) consensus conference on Gleason grading of prostatic carcinoma. Am J Surg Pathol 2005;29:1228-42.

[26] van den Bergh RC, van der Kwast TH, de Jong J, et al. Validation of the novel International Society of Urological Pathology 2014 five-tier Gleason grade grouping: biochemical recurrence rates for $3+5$ disease may be overestimated. BJU Int 2016;118:502-5.

[27] Harding-Jackson N, Kryvenko ON, Whittington EE, et al. Outcome of Gleason $3+5=8$ prostate cancer diagnosed on needle biopsy: prognostic comparison with Gleason $4+4=8$. J Urol 2016;196:1076-81.

[28] Mahal BA, Muralidhar V, Chen YW, et al. Gleason score $5+3=$ 8 prostate cancer: much more like Gleason score 9? BJU Int 2016;118:95-101. 